\title{
Nursing Practice Environment and Its Relation to Missed Nursing Care : A Scoping Review
}

\author{
Rahmah A Aloufi, ${ }^{\star \dagger, 1}$, Maram A Banakhar ${ }^{2}$, Duaa A Hafez ${ }^{3}$ \\ ${ }^{1}$ Faculty of Nursing, King Abdulaziz University, Saudi Arabia \\ ${ }^{2}$ Public Health Department, Faculty of Nursing, King Abdulaziz University, Saudi Arabia \\ ${ }^{3}$ Public Health Department, Faculty of Nursing, King Abdulaziz University, Saudi Arabia
}

DOI https://doi.org/10.15520/ijmhs.v10i07.3059

Accepted 20/07/2020; Received 15/06/2020; Publish Online 28/07/2020

\begin{abstract}
Objective: To examine the association between the practice environment of nursing and the missed care of nursing in a systematic way in the related literature. Methodology: An extensive search was conducted in CINAHL, ProQuest, ERIC, MEDLINE, Web of Science, PubMed, and Science Direct databases. the included studies were published during time period of 2015-2020 with full text. Results: A total of 110 studies were screened, out of which 19 studies were identified to be eligible as per fulfillment of inclusion/exclusion criteria. Degraded quality of nursing, inpatient complications, and mishaps, as well as negative patient outcomes, is examined as examples for results of missed nursing care. The nurses missed the process and task of providing important patient care because of the increased workload, less communication and poor working environment. Inadequate resources and staffing were the major factors that were linked with the events of missed nursing care. Conclusion: It is concluded that reducing and modifying the examined factors, such as poor working environment, inadequate staffing, less resources, poor communication among nurses or patients etc. may decrease the missed nursing care practice. In addition, the missed nursing can also be managed through the practical implementation of motivating positive work environment and safety culture of patients. These improvements and practical implementation will help to provide quality patient care and decrease the missed nursing care.

Keywords: Practice environment, missed nursing care, Teamwork, communication, workload, resources.
\end{abstract}

\section{INTRODUCTION}

The missed nursing care is deliberated as the error omission that takes place when any factor of the needed care of the patient is overdue or delayed $[1,2]$. It is explained in research that around 55 to $98 \%$ of the overall nurses or healthcare workers missed or failed to complete the minimum single activity of care while being on last shifts [3]. In addition, the large number of the continuous missed care of nursing includes different families and patient's education, updating or developing care plans, talking or comforting the patients, oral care and ambulation of the patients, which are considered as the important care element to achieve better patient's safety and outcomes.

* Corresponding author.

† Email: r-d-4@hotmail.com.
The missed care of nursing is defined as the procedure of taking care in the framework of outcomes of the structure process that was suggested by Kim et al., (2018) [4]. Significantly, the framework explained the characteristics influence and organizational structure procedure of care that shows the transaction between the patients and providers throughout the care delivery, and then the care procedures influence the care outcomes. The association related to the missed care in concern of the process of care for the patient results were evaluated in different researches depicting the relationship of missed nursing care with the increased pressure and readmissions injuries resulting in the addition of poorer or worst experience of care of the patient [3, 5].

Significantly, it is highly important to ask which kind of elements and characteristics of the healthcare environment have a major impact on the missed nursing care and require 
improving for the purpose of providing better care outcomes of patient and care quality. In addition, the environment is initially divided in major five dimensions including physician and collegial nurse relation, resource and staffing adequacy, support and leadership of nurses, nurse manager ability, and nursing foundations for care quality [2].

Ball et al., (2018) determined that the factor of missed care is common in some of the hospitals, and the association among the nurse staffing levels and missed care are directly proportional to each other. The term miss care is considered as the care that is rationed care, left undone, or incomplete care. When the levels of registered nurse staffing are less, nursing care is reported to be missed because of the less available time. In addition, the increased missed care rate in turn associates with fewer grades of safety, according to the nurses, patients do not rate their healthcare centers highly and increased fall risk and other negative results can occur [3]. However, it is the consequence of less nurse staffing and potential analyst for the outcomes and experience of patients that can be a basic mechanism because of which the nurse staffing impacts the patient's results. Developing the relationship between missed care and an increase in the death risk of patients is important for considering while implications of patients' safety [4]. If it is mitigated properly, it can increase the safety related to nursing as well [6]. The aim of the review was to examine the literature related to the association between the practice environment of nursing and the missed care of nursing.

\section{MATERIALS AND METHODS}

\section{Search strategy}

An extensive electronic search in multiple databases involving the Cumulative Index Nursing and Allied Health Literature (CINAHL), ProQuest, ERIC, MEDLINE, Web Of Science, PubMed, and Science Direct were used to search for studies that published between 2015 and 2020, applying the limiters "English language" and "Full text". Therefore, it is necessary to review the literature in a systematic way.

The search terms used were "nurse work environment", "nursing practice environment", "nursing work environment", " nurse practice environment "," missed nursing care "," care left undone "and" omitted care". To illustrate how these words were used, the search began with each keyword, and was further developed by gathering and linking terms together by using Boolean operators (AND and OR) to achieve the best literature review possible. Using Boolean operators across all databases allowed the broadening or narrowing of the combined keywords or phrases [7].

In screening of research articles, firstly the abstracts were reviewed for potential eligibility of defined inclusion and exclusion criteria. Only those research studies were selected whom full text was found available. In eight cases of unclear points in study, corresponding author was contacted via email for clarification. By using the keywords targeting each database resulted in many studies. The number of studies identified is shown in the Figure 1.
The inclusion criteria included all study designs that investigate the association between practice environments of nursing and missed care of nursing, examining the association between any factors of practice environment of nursing and missed care of nursing, Published in the English language, published between 2015 and 2020 and Full-text papers.

\section{RESULT}

All the mentioned electronic databases were searched extensively and a total of 110 abstracts were identified using different search terms. From this initial search, 8 studies were removed due to duplication of studies screened from databases. In second phase, 102 abstracts were reviewed, from which 80 of studies were excluded due to not fulfilling the inclusion/exclusion criteria. after careful review of selected 22 research articles, 19 studies were selected who fulfill the inclusion/exclusion criteria and full papers were available for review. A total of four types of studies are included among identified nineteen studies. Cross-sectional studies were found to be most prominent. The distribution of studies is as follows: (a) observational studies $=1$ (b) retrospective studies $=1$ (c) cross-sectional studies $=16(\mathrm{~d})$ mixed-method design $=1$. The maximum number of study participants in a single study was 272,783 while the minimum number of study participants in a single study was 12 nurses.

The included nineteen studies were divided into four main themes, which are: Nursing work environment and missed care, Nurse staffing and missed care, Nurse workload and missed care and Reasons, and factors of missed care.

Nursing Work Environment and Missed Care.

A few studies have shown that there was a connection between missed care and nursing work environment, for example, the cross-sectional study led by Kim et al., (2018) to examine the impact of nursing work circumference and its culture for patient safety practice in the medical center on an instance of missed care. The study sample is made of 188 nurses using a non-probability sample. The study uncovered the significant negative connection between the nursing practice environment and omitted nursing care. Subsequently, the findings of study proposed that through executing mediations that advance a positive nursing workplace and patient safety culture then missed nursing care can be managed [4].

Furthermore, Hessels et al., (2015) conducted a quantitative report in the United States with a random sample consisting of 7,679 nurses to find out the connection present in the nursing working environment and omitted necessary nursing care. The outcomes showed that the practice in the nursing environment is statistically significantly related to missed nursing care [11]. Their discoveries recommended that focused measures to improve the aspects of the nursing workplace will minimize the amount of missed care by nursing [6]. 


\section{Nursing Practice Environment and Its Relation to Missed Nursing Care: A Scoping
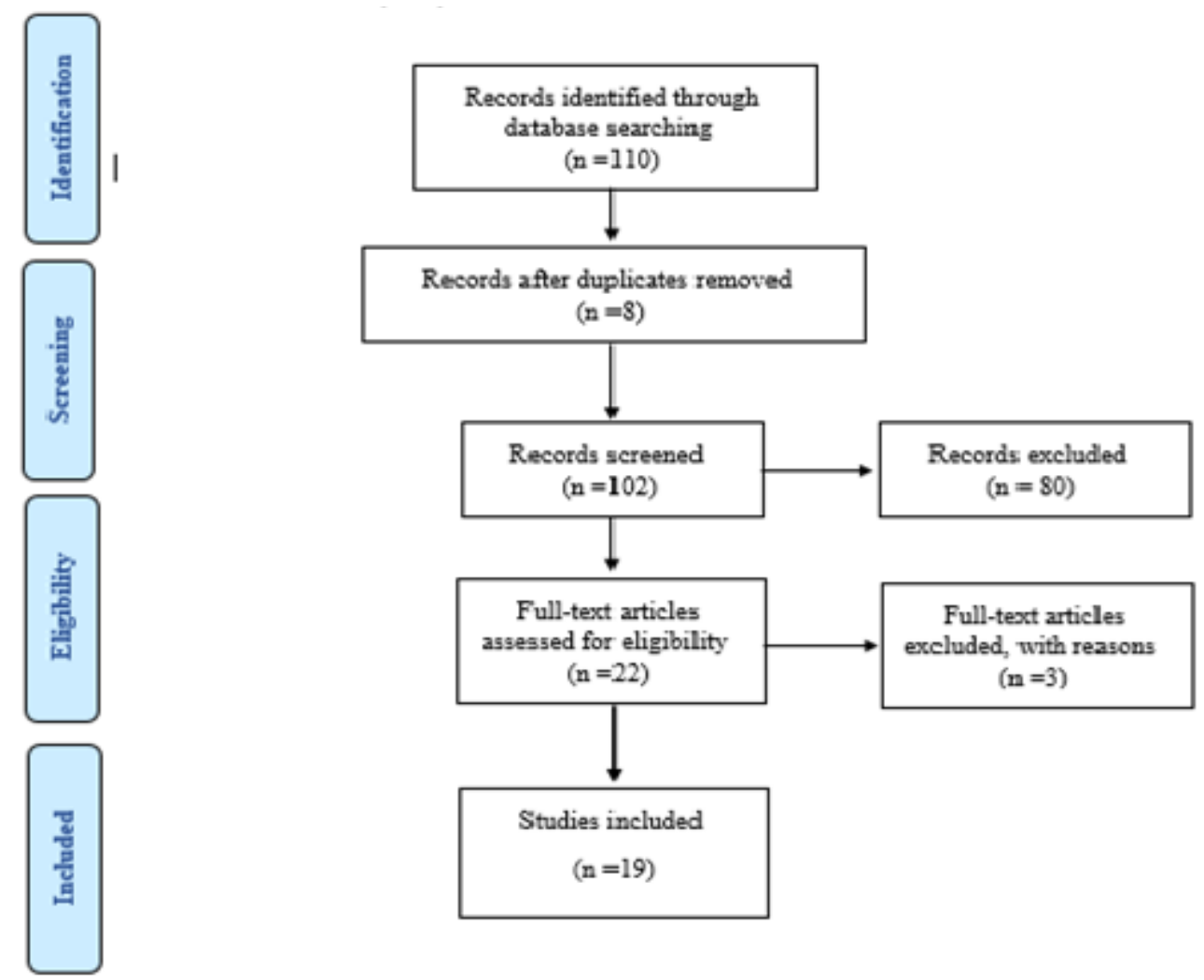

Figure 1: PRISMA of the included

studies

Figure 1.

In accordance with previous studies, Hessels et al., (2015) conducted an additional secondary analysis of crosssectional information in the United States aimed to investigate the connections along with the adoption of Electronic Health Record (EHR) and poor outcomes and satisfaction in hospitalized patients for 7,679 nurses using a random sample. The results of this study present a strong connection linking the nursing environment condition and their missed care [10]. In addition, the study suggested that to improve patient care and the necessary outcomes need for interventions in multi-level [10, 12].

In a further cross-sectional analysis by Carthon et al., (2015) in the United States to investigate the link connecting patient readmission, nursing practice environment, and missed care. The result revealed that the effect of missing nursing care after adjustment to the work environment was not, at this point a significant predictor of readmission. As a result, the study indicated that giving nurses the time and resources to take care of these different needs may prove helpful to reduce readmission. The sample was of 20,605 randomly selected nurses [5].

Likewise, In the United States, Smith et al., (2018) conducted a quantitative cross-sectional study describing the link connecting work environment, collective effectiveness, and the omitted nursing care by surveying 233 nurses. The results showed that one SD increase in the nursing work conditions was significantly linked with 14 less missed care [8]. Several researches have reported that adequate human and material resources to improve team cohesion among staff and reduced missed nursing care [5, 14-16].

Also, Park et al., (2018) conducted a descriptive correlation study to investigate what characteristics of the work environment were linking with missed nursing care in the United States were connected by surveying 31,650 registered nurses. The results uncovered that units with a good environment were $63.3 \%$ lower odds of having missed nursing care than units with a poor environment. Therefore, the study recommended that nursing managers and hospitals need to keep up best practice conditions for nurses to diminish missed nursing care and along these lines possibly improve patient outcomes [2].

In addition, another study by Singh et al., (2019) was conducted in the United States to advance our understanding of how and to what extent nurse burnout affects their delivery of care and the outcome of patients. After concluding the investigation, its outcome indicates that nursing working conditions without subscale for staffing and asset sufficiency correlated negatively with burnout and missed 
care. In addition, staff and resource adequacy correlated poorly with burnout and missed care. In this study, the author used a secondary cross-sectional data analysis for 23,784 registered nurses. As a result, the study proposed that organizational interventions planned to reduce nurse burnout could be an important strategy to relieve costly, occasionally life-threatening adverse events [17]

Furthermore, Blizzard et al., (2018) surveyed 248 nurses in United States to investigate the association between direct-care nurses' viewpoint of implicit rationing of nursing care and their understanding of the leadership style of nurses in the hospital. Their results indicated a huge impact on the nursing practice environment and the implicit rationing of nursing care. In addition, the medium impact for connection between the perspective of better nursephysician relationship and perception of limited implicit rationing of nursing care. Thus, the study recommended that a better nursing workplace maintained by an emotionally intelligent booming leadership style has significant relations with the viewpoint of limited implicit rationing of nursing care by nurses providing direct care [13].

Similarly, cross-sectional study by White et al., (2018) examined the empirical relationship connecting nurse practice environment with outcomes of nurse and patient specifically in a different type of nursing homes. Moreover, the results demonstrated that registered nurses (RNs) who work in better environments of work were $70 \%$ less expected to have missed most of their clinical tasks. The results of this study suggested that several elements of the environment of work are required for assisting RNs to deliver high-quality care in nursing homes [18].

\section{Missed Care and Nurse Staffing}

Numerous researches highlighted the link of nurse staffing with the missed nursing care, for example, Ball et al. (2018) accompanied an observational study in Europe for 26,516 registered nurses to investigate the link present among the nursing staff, mortality, and missed care. In addition, the study found a significant indirect impact, from nursing staffing to missed care and from missed care to mortality. As such, the study suggested that suitable practice and policies workforce are necessary for minimizing the risk of patients dying and missing care from a different type of elements and factors that affect the health system of hospitals, For example, Safe nursing staffing [3].

In accordance with previous studies, Lake et al. (2018) conducted another random sampled retrospective crosssectional study in the United States which is intended to examine the nursing circumstances related to missed nursing tasks in 272,783 registered nurses. The outcome of this research explained that lesser nurse staffing meaningfully raised the likelihood of missed care, while decreased the likelihood is associated with the good nursing working environment. Subsequently, the study suggested that managers should work to increase staffing levels and improve their unit work environment [19].

Azuero et al., (2018) also conducted a study in Australia, in which 1,195 nurses were surveyed to understand the link between socio-demographic characteristics, working environment circumstances, and missed care reports and causes for missed care of nursing. The results that were gathered from the survey enlightened that staff adequacy was the major and one of the direct forecasters of missed care [1].

Furthermore, Zhu et al., (2018) directed a cross-sectional investigation in China with the use of a four-stage sampling for examining the intervention impact of nursing care rationing (RONC) with the addition of the link connecting nursing staff and their patient results for 7802 nurses. Results showed that understaffing in the unit, nurses indicated more RONC. Therefore, the study indicated that a deficiency of nurse staffing prompts RONC, prompting bad patient results [9].

To investigate the association between nurse exhaustion, work capacity, presentism, and safety for patients in United States, a mixed-method study was also conducted [20]. In this study, the author examined 272 registered nurses with the help of a convenience sample. The results showed that over time, which makes up nursing staff and the resulting fatigue, has an overwhelming impact on patient outcomes. In addition, fatigue from overtime led to a mistake in missed care / delayed care. Subsequently, the study indicated that the tiredness of the nurses influenced patient safety and wellbeing, also impacted the nurses themselves $[11,13,20]$.

Nurse Workload and Missed Care

Two studies were found in the literature to investigate the connection linking the workload of nurses and missed care. Furthermore, MacPhee et al., (2017) conducted crosssectional correlation research for almost 472 licensed practice nurses and registered nurses using a stratified random sample to examine the link connecting seven capability determinants with outcomes for patient and nurse in Canada. The study results show that the failure to observe nursing tasks conveyed the effects of a perceived workload on the nursing staff [21]. The recommendation from the finding says that substantial workloads and interruptions influence have a direct and indirect impact on nurse outcomes $[4,13$, 21].

Similarly, Nelson et al., (2017) conducted cross-sectional correlation research for 139 nurses in the United States. Furthermore, the study results showed that the view of the workload correlated significantly positively using the missed nursing care and teamwork of the nurse inversely correlated significantly with the missed care. In addition, the results support that teamwork arbitrate the association of missed nursing care with the workload [12].

\section{Reasons, and Factors of Missed Care}

Several studies also examined the reasons and factors for missed care. For example, a qualitative study by Svolos et al., (2018) was examined for understanding the missed care with the context of a telemetry nurse and for finding out how it continues to occur. The study sample consisted of 12 registered nurses. The study showed that three issues emerged: environmental conditions and nursing staff performance are the two different elements that contribute highly to the missed nursing care, provided the addition of the solution that the use of technology resources can reduce missing nursing care [16]. 


\section{Nursing Practice Environment and Its Relation to Missed Nursing Care: A Scoping Review

Furthermore, Haftu et al. (2019) developed a crosssectional survey in Ethiopia for assessing frequently the elements, factors, and reasons of missed nursing care. Significantly, a total of 401 midwives and nurses randomly taking part in the study. The results of the study showed that material resources, labor resources, communication, and teamwork were identified as causes for common care missing [6].

In addition, the correlated cross-sectional investigation was directed in the United States to explain the connections between hospital structures, processes, and outcomes that reflect the quality and safety of patient care. This result provides nurse administrators present at hospitals with proof on how negative effects of management of nursing upon the negative climate of patient safety and missed nursing care in a healthcare center or hospitals can be addressed [15] [5] [19]. Smith et al., (2016) stated that the incivility of staff and the ability of the nursing manager, the support and leadership of nurses were not a critical pointer of missed care of nursing. An increase in staffing because of the differences present in the prediction of missed care of nursing is also not clinically significant [15].

\section{CONCLUSION}

Based on the search in electronic databases, most of the literature reviewed has identified the different factors connected to the missed nursing care. It is concluded that reducing and modifying the examined factors, such as poor working environment, inadequate staffing, less resources, poor communication among nurses or patients etc. may decrease the missed nursing care practice. In addition, the missed nursing can also be managed through the practical implementation of motivating positive work environment and safety culture of patients, as well as strong leadership and safety. Furthermore, it should be noted that the missed care is not a result synonymous with human abilities but a systemic level of nursing that is influenced by influences in the workforce.

Finally, the literature shows that there is a critical need for further studies that find out the connection linking the working environment for nursing and missed care and how the processes of nurses influence missed nursing care outcomes. So far, no research has explored the association connecting the nursing practice environment with missed care in Saudi Arabia.

Acknowledgement: Great thanks to my supervisors and my family especially my mother and my big brother, also to my friends and colleagues for their support and help.

Conflicts of interest: The authors declared no conflicts of interest.

Funding: No funding availed to conduct this study.

\section{REFERENCES}

[1] A. Azuero, "Likelihood of nursing care being missed is influenced by several work-based factors.," Evidence - Based Nursing, vol. 21, no. 3, p. 68, 2018.
[2] Lake ET, Staiger D, Edwards EM, Smith JG, Rogowski JA. Nursing Care Disparities in Neonatal Intensive Care Units. Health Services Research. 2018;53:30073026. Avail-able from: https:// dx.doi.org/10.1111/1475-6773.12762.।

[3\}. J. E. B. L. Ball, L. H. Aiken, W. Sermeus, D. M. Sloane, A. M. Rafferty and P. Griffiths, "Post-operative mortality, missed care and nurse staffing in nine countries: A cross-sectional study.," International Journal of Nursing Studies, vol. 78, pp. 10-15, 2018.

[4]. K.-J. Kim, M. S. Yoo and E. J. Seo, "Exploring the Influence of Nursing Work Environment and Patient Safety Culture on Missed Nursing Care in Korea," Asian Nursing Research, vol. 12, no. 2, pp. 121-126, 2018. [5]. J. M. B. Carthon, K. B. Lasater, D. M. Sloane and A. KutneyLee, "The quality of hospital work environments and missed nursing care is linked to heart failure readmissions: a crosssectional study of US hospitals.," BMJ quality \& safety, vol. 24, no. $4, \quad$ pp. 255-263, 2015. [6]. M. Haftu, G. Alem, M. Gebremeskel, G. Aregawi, D. Gebregziabher and C. Robles, "Commonly missed nursing cares in the obstetrics and gynecologic wards of Tigray general hospitals;" PLoS One, vol. 14, no. 12, 2019. [7]. D. Polit and C. Beck, "Essentials of nursing research Philadelphia, Baltimore, New York, London, Buenos Aires, Hong Kong, Sydney, Tokyo," Wolters Kluwer, 2018. [8]. J. G. Smith, K. H. Morin, L. E. Wallace and E. T. Lake, "Association of the Nurse Work Environment, Collective Efficacy, and Missed Care," Western journal of nursing research, vol. 40, no. 6, pp. 779-798, 2018. [9]. X. Zhu, J. Zheng, K. Liu and L. You, "Rationing of Nursing Care and Its Relationship with Nurse Staffing and Patient Outcomes: The Mediation Effect Tested by Structural Equation Modeling.," (). International Journal of Environmental Research and Public Health, vol. 16, no. 10, 2019. [10]. A. Hessels, L. Flynn, J. P. Cimiotti, S. Bakken and R. Gershon, "Impact of Heath Information Technology on the Quality of Patient Care.," On-line journal of nursing informatics, vol.

19 ,

2015.

[11]. A. J. Hessels, L. Flynn, J. P. Cimiotti, E. Cadmus and R. R. M. Gershon, "The Impact of the Nursing Practice Environment on Missed Nursing Care.," Clinical nursing studies, vol. 4, no. 3, pp. 60-65, 2015. [12]. S. T. Nelson, "The Relationships Among Workload, Teamwork, and Missed Nursing Care in the Nursing Home Environment.," University of Colorado Denver, Anschutz Medical Campus, Ann Arbor, 2017. [13]. H. L. Blizzard, "The Relationship between the Perceptions of Implicit Rationing of Nursing Care and Emotionally Intelligent Leadership Style among Direct-care Nurses.," Texas Woman's University, 2018. [14]. D. Moher, A. Liberati, J. Tetzlaff and D. G. Altman, "Preferred reporting items for systematic reviews and metaanalyses: the PRISMA statement.," Annals of internal medicine, vol. 151, no. 4, pp. 264-269, 2009. [15]. J. G. Smith, "RN perceptions of coworker incivility and collective efficacy as influential to hospital structures and outcomes," The University of Wisconsin - Milwaukee, 2016. 
[16]. A. M. Svolos, "The State of Missed Nursing Care in a Telemetry Nursing Unit: A Descriptive Case Study.," University of Phoenix, Ann Arbor, 2018. [17]. S. Singh, "The Nexus between Nurse Burnout, Missed Care and Patient Outcomes," University of Pennsylvania, Ann Arbor. , 2019.

[18]. E. M. White, "A Cross-sectional Study to Examine the Effects of Nurse Work Environment on Patient and Nurse Outcomes in Nursing Homes," University of Pennsylvania, Ann Arbor, 2018.

[19]. E. T. Lake, D. Staiger, E. M. Edwards, J. G. Smith and J. A. Rogowski, "Nursing Care Disparities in Neonatal Intensive Care Units.," Health Services Research, vol. 53, pp. 3007-3026, 2018. [20]. C. A. Smith, "Presenteeism in nursing: A mixed methods study of its relationship with nurse fatigue and patient safety.," Mercer University, 2016. [21]. M. MacPhee, V. S. Dahinten and F. Havaei, "The Impact of Heavy Perceived Nurse Workloads on Patient and Nurse Outcomes," Administrative Sciences, vol. 1, no. 7, p. 7, 2017. [22]. Ministry of Health, General Directorate of Statistics \& Information, health statistics annual book, 2013.

\section{AUTHOR BIOGRAPHY}

Rahmah A Aloufi Faculty of Nursing, King Abdulaziz University, Saudi Arabia

Maram A Banakhar Public Health Department, Faculty of Nursing, King Abdulaziz University, Saudi Arabia

Duaa A Hafez Public Health Department, Faculty of Nursing, King Abdulaziz University, Saudi Arabia 